\title{
Reactive control of subsonic axial fan noise in a duct
}

\author{
Y. Liu and Y. S. Choy ${ }^{\text {a) }}$ \\ Department of Mechanical Engineering, The Hong Kong Polytechnic University, Hung Hom, Kowloon, \\ Hong Kong SAR, China \\ L. Huang \\ Department of Mechanical Engineering, University of Hong Kong, Pokfulam Road, Hong Kong SAR, China \\ L. Cheng \\ Department of Mechanical Engineering, The Hong Kong Polytechnic University, Hung Hom, Kowloon, \\ Hong Kong SAR, China
}

(Received 21 May 2014; revised 4 August 2014; accepted 17 August 2014)

\begin{abstract}
Suppressing the ducted fan noise at low frequencies without varying the flow capacity is still a technical challenge. This study examines a conceived device consisting of two tensioned membranes backed with cavities housing the axial fan for suppression of the sound radiation from the axial fan directly. The noise suppression is achieved by destructive interference between the sound fields from the axial fan of a dipole nature and sound radiation from the membrane via vibroacoustics coupling. A two-dimensional model with the flow effect is presented which allows the performance of the device to be explored analytically. The air flow influences the symmetrical behavior and excites the odd in vacuo mode response of the membrane due to kinematic coupling. Such an asymmetrical effect can be compromised with off-center alignment of the axial fan. Tension plays an important role to sustain the performance to revoke the deformation of the membrane during the axial fan operation. With the design of four appropriately tensioned membranes covered by a cylindrical cavity, the first and second blade passage frequencies of the axial fan can be reduced by at least $20 \mathrm{~dB}$. The satisfactory agreement between experiment and theory demonstrates that its feasibility is practical. @ 2014 Acoustical Society of America. [http://dx.doi.org/10.1121/1.4894798]
\end{abstract}

PACS number(s): 43.50.Gf, 43.55.Rg [NX]

Pages: 1619-1630

\section{INTRODUCTION}

Axial-flow cooling fans are extensively used in electronic products like computers and projectors, and in ventilation systems in buildings. The problem of fan noise attracts a lot of attention because noise exposure causes discomfort and annoyance to occupants. The aerodynamic noise generated from a fan is a combination of discrete frequency noise, which is related to the blade passing frequency (BPF), its harmonics, and broadband noise. The basic physical mechanism of fan noise was revealed by Gutin (1948), who studied sound radiation by steady loading on propellers. Such noise radiation is normally insignificant for small fans with a low tip Mach number. Lighthill (1952) established the wellknown "acoustic analogy" as a general theory of aerodynamic sound. This was further extended by Curle (1955) and Ffowcs Williams and Hawkings (1969) to include the effect of all solid boundaries in arbitrary motion. The solid surfaces are regarded as "mirrors" of aerodynamic quadrupoles, giving rise to dipoles distributed all over the solid surfaces which arise from unsteady forces on fluid. These dipole sources are very energetic in radiating sound at low flow speeds. Thus, a real-life fan blade can be modeled as distributed dipole sources along its radial span with a peak strength close to the blade tip due to the usual loading distribution.

\footnotetext{
a) Author to whom correspondence should be addressed. Electronic mail: mmyschoy@polyu.edu.hk
}

Fan noise can be controlled either in the propagating path or directly at the source itself. In the first category, for long ducts, various methods have been developed and adopted based on similar working principles. They all encompass noise reflection by impedance mismatch and/or noise absorption by porous materials or perforated structures (Nijhof et al., 2004a,b; Beranek and Ver, 2006; Munjal, 1987). For short ducted fans, the choices are limited. The common approach consists of using a duct liner with a uniform (Baumeister,1979) or tandem non-uniform layer of porous material with a compliant structure under optimized flow resistivity of the material (Eversman, 2001) or perforated panel absorbers (Wu, 1997; Maa, 1998). The sound absorption capability of this kind of liner or absorber is restricted by its length and depth compared with the long wavelength of low frequency noise. The active noise control method is an effective way of controlling the low frequency component. It is used along the propagating path by virtue of the array of actuators to produce the cancelling sound field. These array actuators are composed of an array of microphones (Koopmann et al., 1988; Gerhold, 1997), resonant type drivers (Walker, 1999), or conventional electromagnetic drivers (Lauchle et al., 1997). However, the issues of cost, sophistication of the design, and reliability have so far prevented this technology from finding widespread use in engineering.

Previous work in the area of direct control on the fan mainly includes blade-vane count selection to reduce the rotor-stator interaction tone noise, rotor-stator spacing optimization to weaken the impinging wakes, and clean inlet 
designs to minimize inflow distortion on the blade (Envia, 2001). For instance, Kameier and Neise (1997) put a turbulence generator into the tip clearance gap to reduce the broadband tip clearance noise. Some researchers have used guide vane sweep and lean with an increase of the spacing between the rotor and stator to reduce fan noise. The achieved noise reduction is due to additional viscous wake decay and additional variation that occurs in the phase of the incident wave along the vane span (Envia, 2001).

However, little attention has been devoted to the direct control of the fan noise source itself for reducing the sound radiation capability without modifying the fan configuration. Recently, the use of flow control obstructions to control tonal noise from a subsonic axial fan was proposed. Significant noise attenuation was obtained by putting different optimized obstructions at the upstream side of the fan with very small percentage loss of the flow capacity (Gerard et al., 2009, 2012). The working principle of that technique is based on the destructive interference between the original unsteady lift and secondary unsteady lift due to the obstruction. In order to eliminate the blockage of the flow due to the use of obstructions, Huang et al. (2010) proposed another passive method by creating destructive acoustic interference between the sound fields at two sides of the dipole fan noise source. However, such a configuration with a sudden change of cross-sectional area will decrease the air flow through the duct, resulting in an unavoidable pressure loss.

From another point of view, the airflow generated by the fan usually causes extra problems that deteriorate the performance of the silencers in the ductwork system. For example, airflow creates noise associated with turbulent flow and flow separation as flow borne sound when the duct changes dimensions or direction. Structural vibration can also be induced as a result of the coincidence of the vortex shedding with the acoustical resonance (Parker, 1972). In addition, pressure loss and flow blockage exist in most designs due to the changes stated above. To eliminate the back pressure and ensure the air flow status, a thin membrane can be used to cover an expansion chamber, which is the so-called tensioned membrane housing. The performance of this device to control noise radiation from the dipole source without flow has been studied analytically and validated by experiment using loudspeakers (Liu et al., 2012). In fact, the radiated sound from a ducted fan has an anti-phase relation towards the two sides and roughly behaves like a dipole source. Hence, the current study is a step forward in the investigation of the performance of this device for actual fan operation. In the case of fan operation, there is interaction between the rotating blade and stator, tip leakage flow, and turbulence on the blade and duct wall. With the fan approaching the tensioned membrane housing, apart from deforming the membrane due to the pressure difference when the fan rotates at high speed, there is very strong fluidstructure and acoustic coupling. As a simplification, the flow effect is divided into two parts: the effect of uniform flow on the capability to undergo sound cancellation and turbulent flow on the vibration of the membrane. The mean flow effect on a drum silencer, consisting of two cavity-backed membranes on the duct walls installed downstream of the fan, has been investigated (Choy and Huang, 2005). It was found that turbulence-induced vibration was insignificant when the flow mach number is small $(M<0.05)$ and the main effect of flow is mainly to deteriorate the silencer performance due to the existence of the cross-modal coupling which is absent without flow. For the current device with a fan housed by the cavitybacked membranes, the sound waves from the fan of a dipole nature will radiate waves of two different speeds due to the convective effect in the case of mean flow. This leads to asymmetric excitation loading on the membrane. The objectives of the present study are (1) to establish a theoretical vibroacoustic model, which takes into account the full coupling between the membrane vibration, asymmetrical sound radiation from the dipole, and the sound fields in the duct with the mean flow effect and acoustic waves inside the cavity, (2) to understand the sound cancellation mechanism with the convective effect as well as its impact on silencing performance, and (3) to investigate the appropriate location of the sound source to cope with the asymmetrical loading effect of the flow.

In what follows, Sec. II proposes a 2D analytical model in detail. In Sec. III, a numerical simulation and experimental study are conducted to validate the theoretical model. In Sec. IV, the performance analysis including the response of the membrane and radiation waves are discussed. Finally the main conclusions are summarized in Sec. V.

\section{ANALYTICAL MODELING OF 2D CONFIGURATION}

Figure 1 shows the two-dimensional configuration of the proposed device, which is composed of two flush-mounted membranes covering a side-branch rigid-walled cavity in an otherwise rigid-walled duct with a height $h$. Membranes of length $L$ are supported at both the leading $(x=-L / 2)$ and trailing edges $(x=L / 2)$, and tension $T$ is applied along the axial direction. For convenience, all parameters are normalized by a set of three basic quantities: air density $\rho_{0}$, speed of sound $c_{0}$, and the duct height $h$. So, the frequency $f$ is normalized by $c_{0} / h$, tensile force per unit length $T$ by $\rho_{0} c_{0}^{2} h$, membrane mass per unit area $m$ by $\rho_{0} h$, and the flow speed $U$ by $c_{0}$. An axial fan of dipole nature is placed at the center of the duct. Dipole sound waves $p_{d}$ from the fan are radiated from $x=x_{s}$ to both the upstream and downstream sides with unit amplitude of the concentrated force exerted on the fluid by the dipole source and with the mean flow speed $M=U / c_{0}$, expressed as (Rodarte and Miller, 2001)

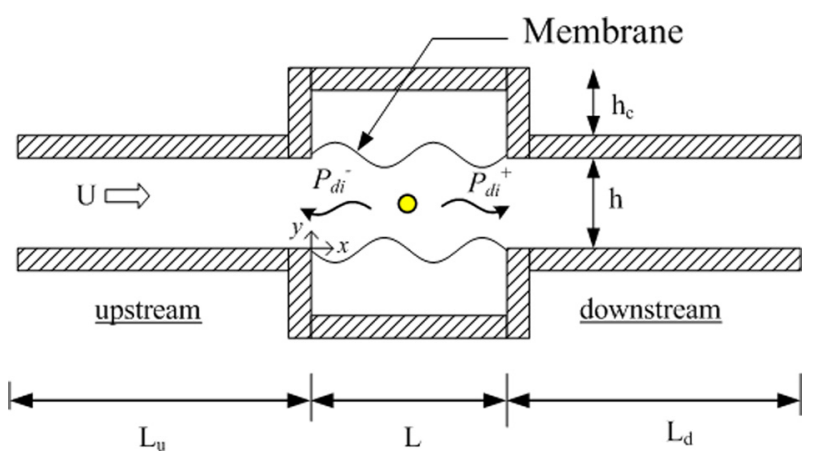

FIG. 1. (Color online) Two-dimensional model of an axial fan inside an expansion chamber with two tensioned membranes flush mounting on the wall. 


$$
\begin{aligned}
p_{d}= & \sum_{m=0}^{\infty} \frac{\left(2-\delta_{0 m}\right) \cos \left(m \pi y_{s}\right) \cos (m \pi y)}{\left(1-M^{2}\right)\left(k_{m}^{+}+k_{m}^{-}\right)} \\
& \times\left[k_{m}^{+} e^{-i k_{m}^{+}\left(x-x_{s}\right)} H\left(x-x_{s}\right)-k_{m}^{-} e^{i k_{m}^{-}\left(x-x_{s}\right)} H\left(x_{s}-x\right)\right],
\end{aligned}
$$

where $k_{m}^{ \pm}=\mp k_{0} M-i \sqrt{\left(1-M^{2}\right)(m \pi / h)^{2}-k_{0}^{2}} /\left(1-M^{2}\right)$.

The membrane vibration with the displacement $\eta$ is driven by the air pressure difference across the interface at $y=0$,

$$
m \frac{\partial^{2} \eta}{\partial t^{2}}-T_{x} \frac{\partial^{2} \eta}{\partial x^{2}}+\left(p_{d}+p_{\text {vib }}-p_{\text {cav }}\right)=0
$$

where $T_{x}$ is the tensile forces applied in the axial direction respectively, and $p_{\mathrm{vib}}$ and $p_{\mathrm{cav}}$ are the fluid loading on the upper $\left(y=0^{+}\right)$and lower sides $\left(y=0^{-}\right)$of the membrane induced by the membrane vibration itself, respectively. Structural damping in such a membrane is assumed to be very small and is ignored. Two identical membranes are simplified to a problem in which one membrane is installed in a duct at $y=0$ and the symmetric plane of $y=h / 2$ is replaced by a solid wall. With this simplification, the presence and the effect of the upper membrane at $y=h$ are ignored in the subsequent theoretical development. In order to solve Eq. (2), both membrane vibration and fluid loading $\Delta p=p_{\text {vib }}-p_{\text {cav }}$ on the membrane are expanded into the in vacuo modes denoted by

$$
V(x, t)=e^{i \omega t} \sum_{j=1}^{\infty} V_{j} \sin (j \pi \xi)
$$

where $\xi=(x / L+1 / 2)$ is the local dimensionless variable, and

$$
\Delta p=\sum_{j=1}^{\infty} V_{j} p_{j}^{1}
$$

where $p_{j}^{1}$ is the fluid loading caused by the modal vibration of unit amplitude of $V_{j}$.

The sound in the duct is coupled by a kinematic boundary condition on the membrane surface from the membrane $\left(y=0^{+}\right)$with the vibration velocity, $V_{M}=i \omega \eta+M(\partial h / \partial x)$. For a duct of infinite length, the radiated sound caused by the vibration of the membrane in the duct is (Doak, 1973)

$$
p_{\mathrm{vib}}=\frac{i L}{2} \sum_{m=0}^{\infty} \frac{\psi_{m}(y) \psi_{m}\left(y^{\prime}\right)}{\sqrt{\left(1-M^{2}\right)(m \pi)^{2}-k_{0}^{2}}} \int_{0}^{1}\left[H\left(x-x^{\prime}\right)\left(k_{0}-M k_{m}^{+}\right) e^{-i k_{m}^{+}\left(x-x^{\prime}\right)}+H\left(x^{\prime}-x\right)\left(k_{0}+M k_{m}^{+}\right) e^{i k_{m}^{-}\left(x-x^{\prime}\right)}\right] V_{M} d \xi^{\prime}
$$

where $\psi_{m}(y)=\sqrt{2-\delta_{0 m}} \cos (m \pi y / h), H$ is the Heaviside function, and $\delta_{l j}$ is the Kronecker delta function.

In this regard, the modal impedance derives from $p_{\text {vib }}$ which is now given by

$$
Z_{\mathrm{vib}, j l}=\left.2 \int_{0}^{1} p_{\mathrm{vib}}\right|_{j} \sin (l \pi \xi) d \xi
$$

where subscript $j$ refers to the membrane vibration mode while $l$ is the resulting pressure coefficient.

The modal impedance $Z_{\mathrm{vib}, j l}$ can be divided into two parts: one is attributed to the radiated pressure from the membrane, denoted by $\left(Z_{\mathrm{vib}, L, j l}\right)$, and another one is attributed to the convective effect that is denoted by $Z_{\mathrm{vib}, C, j l}$, expressed as

$$
Z_{\mathrm{vib}, j l}=Z_{\mathrm{vib}, L, j l}+Z_{\mathrm{vib}, C, j l},
$$

where

$$
\begin{aligned}
Z_{\mathrm{vib}, L, j l}= & \frac{2\left(k_{0}-M k_{m}^{+}\right)}{(j \pi)^{2}-\left(k_{m}^{+} L\right)^{2}}\left(\frac{j l \pi^{2}\left(1-e^{-i k_{m}^{+} L} \cos l \pi\right)}{(l \pi)^{2}-\left(k_{m}^{+} L\right)^{2}}+\frac{j l(1-\cos j \pi \cos l \pi)}{j^{2}-l^{2}}\right) \\
& +\frac{2\left(k_{0}+M k_{m}^{-}\right)}{(j \pi)^{2}-\left(k_{m}^{-} L\right)^{2}}\left(\frac{j l \pi^{2} \cos j \pi\left(\cos l \pi-e^{\left.-i k_{m}^{-} L\right)}\right.}{(l \pi)^{2}-\left(k_{m}^{-} L\right)^{2}}-\frac{j l(1-\cos j \pi \cos l \pi)}{j^{2}-l^{2}}\right)
\end{aligned}
$$

and

$$
\begin{aligned}
Z_{\mathrm{vib}, C, j l}= & \frac{-2 j \pi M}{L \omega}\left\{\frac{\left(k_{0}-M k_{m}^{+}\right) k_{m}^{+} L}{\left((j \pi)^{2}-\left(k_{m}^{+} L\right)^{2}\right)}\left(\frac{l(1-\cos (j \pi) \cos (l \pi))}{\left(l^{2}-j^{2}\right) \pi}-\frac{l \pi\left(1-e^{-i k_{m}^{+} L} \cos (l \pi)\right)}{l^{2} \pi^{2}-k_{m}^{+2} L^{2}}\right)\right. \\
& \left.+\frac{\left(k_{0}+M k_{m}^{-}\right) k_{m}^{-} L}{(j \pi)^{2}-\left(k_{m}^{-} L\right)^{2}}\left(\frac{l(1-\cos (j \pi) \cos (l \pi))}{\left(l^{2}-j^{2}\right) \pi}+\frac{l \pi \cos (j \pi)\left(\cos (l \pi)-e^{-i k_{m}^{-} L}\right)}{l^{2} \pi^{2}-k_{m}^{-2} L^{2}}\right)\right\} .
\end{aligned}
$$


Similarly, the acoustic pressure inside a cavity with the length $L_{c}$ and height $h_{c}$ acting on the membrane $\left(y=0^{-}\right)$ can be obtained through the cavity mode decomposition (Kuttruff, 2000).

$$
\begin{aligned}
p_{\text {cav }}(x, y)= & \sum_{r, s}^{\infty} \frac{-i \omega \phi_{r s}(x, y)}{L_{c} h_{c}\left(\kappa_{r, s}^{2}-k_{0}^{2}+2 i \zeta_{r, s} \kappa_{r, s} k_{0}\right)} \\
& \times \int_{0}^{L_{c}} V\left(x^{\prime}, 0\right) \phi_{r s}\left(x^{\prime}, 0\right) d x^{\prime}
\end{aligned}
$$

where $\zeta_{r, s}$ is the damping ratio of the $(r, s)$ th acoustic mode $\phi_{r s}(x, y)$ and $\kappa_{r, s}$ is the corresponding acoustic wave number, given as

$$
\begin{aligned}
& \phi_{r s}(x, y)=\sqrt{\left(2-\delta_{0 r}\right)\left(2-\delta_{0 s}\right)} \cos \left(\frac{r \pi x}{L_{c}}\right) \cos \left(\frac{s \pi y}{h_{c}}\right), \\
& \kappa_{r, s}=\sqrt{\left(\frac{r \pi}{L_{c}}\right)^{2}+\left(\frac{s \pi}{h_{c}}\right)^{2}} .
\end{aligned}
$$

Hence,

$$
Z_{\mathrm{cav}, j l}=2 \int_{0}^{1} p_{\mathrm{cav}}(x, 0) \sin (l \pi \xi) d \xi=\sum_{r, s}^{\infty} \frac{-2 i k_{0} L\left(2-\delta_{0 r}\right)\left(2-\delta_{0 s}\right)}{L_{c} h_{c}\left(\kappa_{r, s}^{2}-k_{0}^{2}+2 i \zeta_{r, s} \kappa_{r, s} k_{0}\right)}\left[\frac{l \pi(1-\cos l \pi \cos r \pi) j \pi(1-\cos j \pi \cos r \pi)}{(r \pi)^{2}-(l \pi)^{2}} \frac{r \pi)^{2}-(j \pi)^{2}}{(r \pi} .\right.
$$

Having found the modal radiation and cavity reflection, Eq. (2) can be expressed as a set of linear equations for modal vibration amplitude, $V_{j}$,

$$
L_{j} V_{j}+\sum_{j l}\left[Z_{\mathrm{vib}, j l}+Z_{\mathrm{cav}, j l}\right] V_{j}=-I_{d}
$$

where $L_{j}=T_{x} / i \omega(j \pi / L)^{2}+m i \omega$ and $I_{d}=2 \int_{0}^{1} p_{d} \sin (l \pi \xi) d \xi$.

As a result,

$$
\begin{aligned}
I_{d}= & \sum_{m=0} \frac{-\left(2-\delta_{0 m}\right) k_{m}^{-} F_{x} \cos \left(k_{y m} y\right) \cos \left(k_{y m} y_{s}\right)}{\left(1-M^{2}\right)\left(k_{m}^{+}+k_{m}^{-}\right)}\left(\frac{l \pi e^{-i k_{m}^{-} \xi_{s} L}-l \pi \cos \left(l \pi \xi_{s}\right)+i k_{m}^{-} L \sin \left(l \pi \xi_{s}\right)}{l^{2} \pi^{2}-k_{m}^{-2} L^{2}}\right) \\
& +\sum_{m=0} \frac{\left(2-\delta_{0 m}\right) k_{m}^{+} F_{x} \cos \left(k_{y m} y\right) \cos \left(k_{y m} y_{s}\right)}{\left(1-M^{2}\right)\left(k_{m}^{+}+k_{m}^{-}\right)}\left(\frac{l \pi \cos \left(l \pi \xi_{s}\right)+i k_{m}^{+} L \sin \left(l \pi \xi_{s}\right)-l \pi e^{-i k_{m}^{+} L\left(1-\xi_{s}\right)} \cos l \pi}{l^{2} \pi^{2}-k_{m}^{+2} L^{2}}\right) .
\end{aligned}
$$

The sound pressure propagating towards both upstream and downstream exits at the far field can be expressed by taking only the plane wave mode for low frequencies for Eqs. (4) and (1). The sound pressure at the upstream and downstream exits is expressed as

$$
p_{\text {upexit }}=\frac{\left.p_{\mathrm{vib}}(x, y)\right|_{m=0, x \rightarrow-\infty}}{e^{i k_{m}^{-} x}}+\left.p_{d}(x, y)\right|_{m=0, x \rightarrow-\infty}=\sum_{j=1}^{\infty} V_{j} \frac{j \pi L e^{i k_{0}^{-} L / 2}\left(1-e^{-i k_{0}^{-} L} \cos j \pi\right)}{2(1-M)\left((j \pi)^{2}-\left(k_{0}^{-} L\right)^{2}\right)}\left(1+\frac{M k_{0}^{-}}{\omega}\right)-\frac{k_{0}^{-} e^{-i k_{0}^{-} x_{s}}}{\left(1-M^{2}\right)\left(k_{0}^{+}+k_{0}^{-}\right)}
$$

and

$$
\begin{aligned}
p_{\text {downexit }} & =\frac{\left.p_{\mathrm{vib}}(x, y)\right|_{m=0, x \rightarrow+\infty}}{e^{i k_{m}^{+} x}}+\left.p_{d}(x, y)\right|_{m=0, x \rightarrow+\infty} \\
& =\sum_{j=1}^{\infty} V_{j} \frac{j \pi L e^{-i k_{0}^{+} L / 2}\left(1-e^{i k_{0}^{+} L} \cos j \pi\right)}{2(1+M)\left((j \pi)^{2}-\left(k_{0}^{+} L\right)^{2}\right)}\left(1-\frac{M k_{0}^{+}}{\omega}\right)+\frac{k_{0}^{+} e^{i k_{0}^{+} x_{s}}}{\left(1-M^{2}\right)\left(k_{0}^{+}+k_{0}^{-}\right)},
\end{aligned}
$$

respectively.

In the current study, an insertion loss (IL) defined as the level difference of the acoustical power radiated from the unsilenced and silenced system, is considered. The unsilenced system is a straight duct with the same uniform cross-section area $s$ and total length $L$. The dipole source is kept at the same position in the duct. Thus the total IL at the two exit boundaries at the downstream and upstream is calculated as 


$$
\begin{aligned}
& \mathrm{IL}=10 \log _{10}\left(\frac{\left.\int p_{d}\right|_{m=0, x \rightarrow-\infty}{ }^{2} d s}{\int p_{\text {upexit }}^{2} d s}\right) \text { and } \\
& \mathrm{IL}=10 \log _{10}\left(\frac{\left.\int p_{d}\right|_{m=0, x \rightarrow+\infty}{ }^{2} d s}{\int p_{\text {downexit }}^{2} d s}\right),
\end{aligned}
$$

respectively.

\section{NUMERICAL AND EXPERIMENTAL VALIDATIONS}

Both a numerical approach and experimental investigations have been adopted to validate the analytical predictions. Apart from validating the mathematical model in $2 \mathrm{D}$, the numerical approach with finite element simulation has the advantage of offering wide applications of the complicated geometry or configuration in $2 \mathrm{D}$ or $3 \mathrm{D}$ with flow for practical use. The experimental validation is also important in order to realize the proposed device for silencing axial fan noise by destructive interference of the sound wave with an anti-phase relationship.

\section{A. Numerical simulation}

To solve the problem numerically, the commercial software COMSOL MULTIPHYSICS ${ }^{\circledR}$ finite element method (FEM) was employed because of its strong multiphysics capability. The numerical simulation has two domains. One is the acoustical domain for fluid to describe the sound wave propagation in the flow duct and cavity. The cavity and the duct are geometrically disconnected. Another is the structural domain for the induced vibration of the membrane. The fluid inside the flow duct is assumed to be uniform, inviscid, perfectly isentropic and irrotational. The unsteady fluid dynamics of the air in the duct in 3D configuration is governed by the convective wave equation with acoustic pressure,

$$
\begin{aligned}
& \left(1-M^{2}\right) \frac{\partial^{2} p}{\partial x^{2}}+\frac{\partial^{2} p}{\partial y^{2}}+\frac{\partial^{2} p}{\partial z^{2}}-\frac{2 i \omega M}{c_{0}} \frac{\partial p}{\partial x}+\frac{\omega^{2}}{c_{0}^{2}} p \\
& =\frac{\partial F_{x}}{\partial x} \delta\left(x-x^{\prime}\right) .
\end{aligned}
$$

The dynamic of the membrane in $2 \mathrm{D}$ is governed by the following equation:

$$
m(i \omega)^{2} \eta-T_{x} \frac{\partial^{2} \eta}{\partial x^{2}}-T_{z} \frac{\partial^{2} \eta}{\partial z^{2}}+\left(\left.p\right|_{y=0+}-\left.p\right|_{y=0-}\right)=0
$$

where $\left.p\right|_{y=0+}-\left.p\right|_{y=0-}$ is the sound pressure difference across the interface $y=0^{+}$.

The sound pressure in the duct is coupled through a kinematic boundary condition on the upper membrane surface $(y=0+)$. The boundary condition of the fluid domain at $y=0+$ is given by the vertical momentum equation

$$
\rho_{0}\left(i \omega V_{M}+M \frac{\partial V_{M}}{\partial x}\right)+\frac{\partial p}{\partial y}=0 .
$$

Hence,

$$
\left.\frac{\partial p}{\partial y}\right|_{y=0+}=-\rho_{0}\left(i \omega V_{M}+M \frac{\partial V_{M}}{\partial x}\right)
$$

For the frequency below the first cut-on frequency of the duct, two exit boundaries at the upstream and downstream can be expressed as

$$
\frac{\partial p}{\partial n}+\frac{Z^{-1} i k p}{(1-M)}=0 \quad \text { and } \quad \frac{\partial p}{\partial n}+\frac{Z^{-1} i k p}{(1+M)}=0,
$$

respectively, where the outward normal direction $n$ coincides with $x$ and $Z$ is the open end impedance of the duct with a correction factor for flow effect. For an unflanged duct with equivalent radius $a$ (Ville and Richard, 1980),

$$
Z=\frac{1+\sigma \beta}{1-\sigma \beta}, \quad \sigma=\left(\frac{1-M}{1+M}\right)^{1.33}, \quad \beta=\frac{Z_{0}-1}{Z_{0}+1}
$$

where $Z_{0}=\frac{1}{4}(k a)^{2}+j k \delta_{0}, \delta_{0}=0.6133 a$ (Atig et al., 2004). For outgoing waves, no reflection occurs thus impedance is 1. Other walls of the duct and cavity are assumed to be acoustically rigid.

\section{B. Comparison between numerical simulation in 2D configuration and analytical result}

In order to compare with the analytical solution in the 2D configuration, the FEM model in the 3D configuration described in Sec. III A is converted into a $2 \mathrm{D}$ model by removing one of the directions. The comparisons are made with analytical solutions for the membrane with dimensionless mass ratio $m=0.4$, dimensionless tension $T_{x}=0.05$ and source location $x_{s}=0.06$. Figures 2(a) and 2(b) compare the IL spectrum between the analytical prediction (circle and dashed line) and FEM simulation model in 2D (solid line) at the upstream and downstream sides, respectively. In the
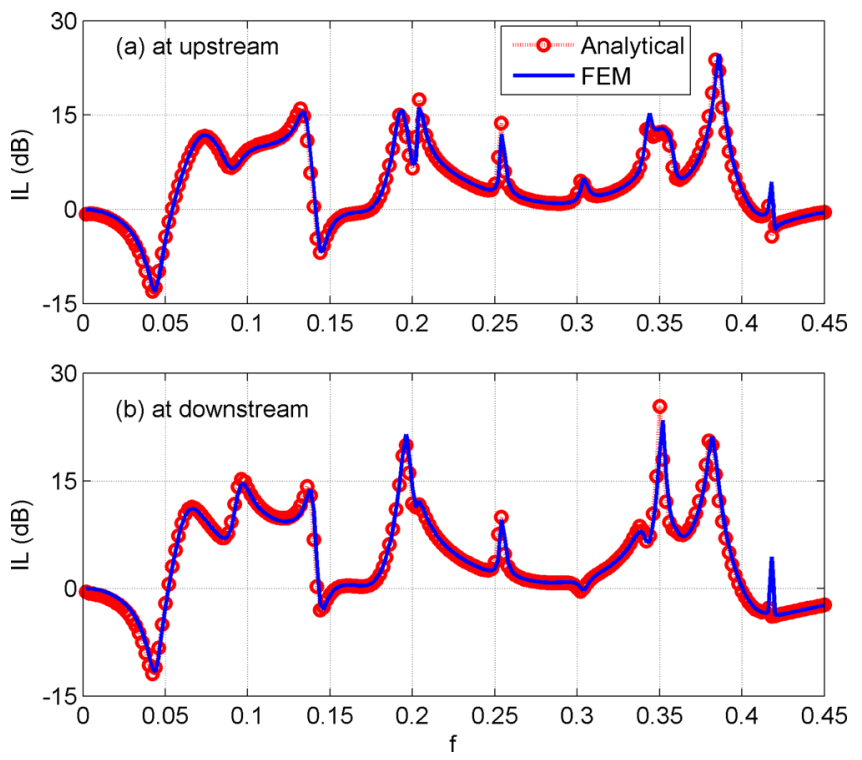

FIG. 2. (Color online) Comparison between the numerical and analytical IL in cases of flow ( $m=0.4, T=0.05, x_{s}=0.06$ ) (a) upstream and (b) downstream. 


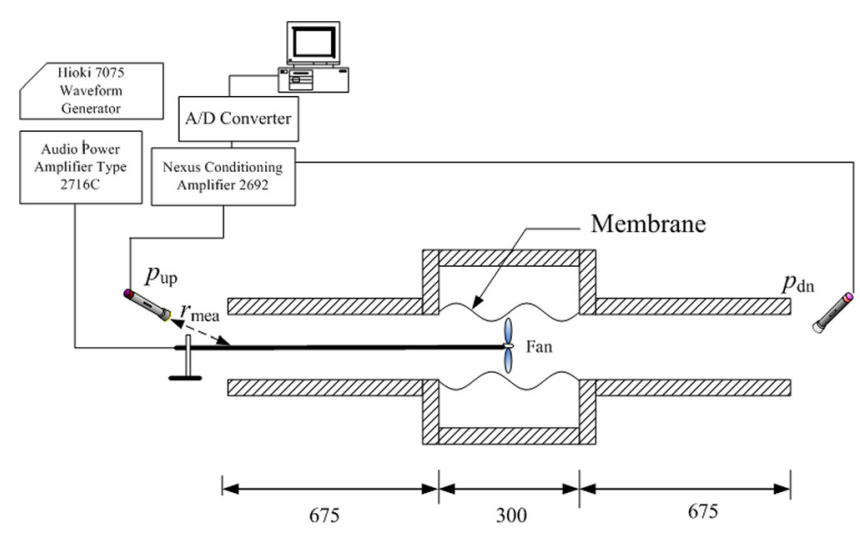

FIG. 3. (Color online) Experimental set-up for extraction pure dipole sound pressure level (SPL) and IL.

numerical simulation, a total of around 17000 Lagrangian, quadratic, triangular elements are used for the fluid domain and 101 nodes are used for the one-dimensional membrane. In the analytical calculation, the duct mode number is truncated as 30 and the membrane's vibration mode is 25 . The FEM simulation matches the analytical results quite well, and no visible deviation between the results from the two methods can be observed except for minor frequency shifts around the spectral peaks. In the design of the proposed device, the main concern was the stopband for covering the first and second blade passage frequency and the resonant peaks were of little concern. In light of this, this finite element simulation was regarded as sufficiently accurate for the purpose of this study. Therefore, the numerical tool is capable of being extended to handle similar problems with complicated configurations.

\section{Experimental validation}

The experimental setup is illustrated in Fig. 3. The rectangular duct wall is made of $15 \mathrm{~mm}$ thick acrylic and is considered to be acoustically rigid. The cross-section of the main duct is $100 \mathrm{~mm} \times 100 \mathrm{~mm}$. The first cut-on frequency in the rigid duct is about $1700 \mathrm{~Hz}$ which is higher than the upper limit of the measured frequency range. The length of the two side-branch cavities is $300 \mathrm{~mm}$ which is the same as that of the membrane and its depth is $200 \mathrm{~mm}$. The upstream and downstream ducts connecting with the leading and trailing edge of the membrane, respectively, are $675 \mathrm{~mm}$ long. The membrane is made of stainless steel (Precision Brand ${ }^{\circledR}$ ), $300 \mathrm{~mm}$ long, $100 \mathrm{~mm}$ wide, and $0.025 \mathrm{~mm}$ thick. The density and Young's modulus are $7.8 \mathrm{~g} / \mathrm{cm}^{3}$ and $193 \mathrm{GPa}$, respectively. Hence the corresponding mass ratio is about 1.4. The membranes are clamped and stretched by a special designed tensile machine (Choy and Huang, 2002). The tensile force applied is measured by a strain gauge glued to the surface of the membrane in the test section. The strain gauge sensor (FLA-3-11-1Lt) has dimensions of $6 \mathrm{~mm} \times 3 \mathrm{~mm}$ and its attachment does not have a noticeable influence on the dynamics of the stretched membrane. In order to minimize the acoustical leakage and flow instability, the lateral edges are then glued and fixed by a very thin tape along the axial direction to seal the gap and no tension is applied on the membrane in the lateral direction. In addition, an axial fan with a rotor $82.5 \mathrm{~mm}$ in diameter with an outer frame with the dimensions $92 \mathrm{~mm} \times 92 \mathrm{~mm} \times 38 \mathrm{~mm}$ is placed at the center of the duct. The fan assembly is held as a cantilever by a very rigid rod and the position of the fan is adjustable along the axial direction by extending the length of this rod. The flow velocity is measured by traversing a hot wire probe across the inlet section of the duct and for the axial fan is found to be $3.6 \mathrm{~m} / \mathrm{s}$. The radiated noise is measured both at $20 \mathrm{~cm}$ upstream and the downstream outlet of the duct with an angle of $\theta=30^{\circ}$ from the near-side duct opening plane. The operation of the fan is controlled by a regulated power supply and consequently sound power of the fan can also be varied. One pair of $1 / 2$ in. microphones B\&K type 4189 with windscreen are used and supported by Nexus Conditioning Amplifier type 2693. The signals from the microphones are acquired through an AD converter (NI DAQ Card 6062E) and the data acquisition process is controlled by the MATLAB program. Finally the sound pressure directly measured at the exits of the upstream and downstream duct are denoted by $p_{\text {up }}$ and $p_{\mathrm{dn}}$, respectively. Practically, the length of the duct is limited and then the sound radiated from one open end of the duct can reach the microphone located on the other side but the cross influence is assumed to be very small. On the other hand, in reality, the radiation from the fan is not an ideal dipole. There is a need to extract the dipole component from the real noise source during the signal processing. The radiated sound approaching the two openings is a plane wave when the duct is long enough. Reflected sound in the case of flow is also scattered by the fan blades and the junctions between the interface at the rigid wall and flexible membrane. Ultimately, this sound will also be combined with the radiated sound from membranes which are induced into vibration. Technically, it is very difficult to decompose all wave components in such a complex environment for the coupled system. Nevertheless, it is possible to model the whole coupled configuration of the cavity-backed membrane and the fan as an equivalent source. The sound wave decomposition procedure (Huang et al., 2010) is modified here when there is flow. The sound radiation of the noise source is considered to consist of two components: one dipole $p_{d}$ with an anti-phase relation in the upstream and downstream regions, and another monopole $p_{m}$ with an in-phase relation. Assuming that the fan is located at the origin,

$$
p=\left\{\begin{array}{l}
\left(p_{m}+p_{d}\right) e^{+i k_{0}^{-} x}+B e^{+i k_{0}^{-} x}+C e^{-i k_{0}^{+} x}, x<-L / 2, \\
\left(p_{m}-p_{d}\right) e^{-i k_{0}^{+} x}+B e^{+i k_{0}^{-} x}+C e^{-i k_{0}^{+} x}, x>+L / 2,
\end{array}\right.
$$

where $B$ and $C$ are the reflections of the equivalent source radiation by the duct ends. Having done the decomposition, the corresponding $\mathrm{IL}$ for the net dipole radiation can be obtained,

$$
\mathrm{IL}_{d}=10 \log _{10} \frac{\left|p_{d}\right|^{2} \text { original }}{\left|p_{d}\right|^{2} \text { silenced }} .
$$

For validation purposes only, $T=0.2$ (the dimensional tensile force: $300 \mathrm{~N}$ ) is applied to the membrane in the current 

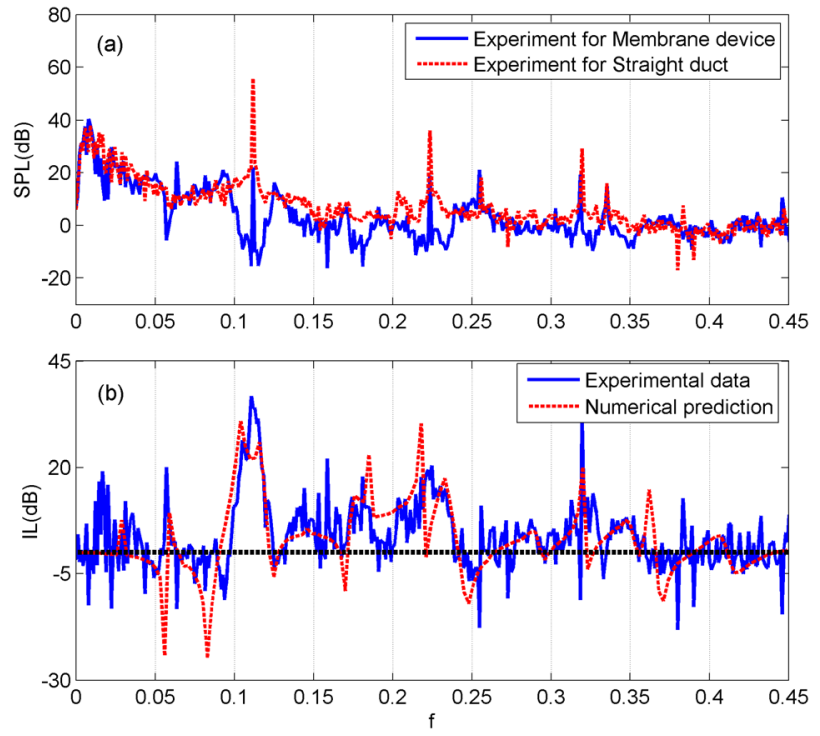

FIG. 4. (Color online) Experimental results for the axial flow fan housed by the membrane. (a) SPL. (b) IL downstream. $\left(T=300 N, x_{s}=0, M=0.015\right.$.)

experiment. Figure 4(a) shows that the fan noise spectrum without silencing treatment measured downstream has sharp peaks which are attributed to blade passing events (dashed line). With silencing by two tensioned membranes with cavities (solid line), the sharp peaks diminish significantly and the SPL in the frequency range of $f=0.094$ to $f=0.124$ is also decreased. The IL under the extraction scheme measured downstream IL is shown in Fig. 4(b) as a solid line, which is compared with the numerical prediction by considering the open end condition (dashed line). The agreement between them is generally satisfactory in terms of the overall IL level and the spectral pattern. The IL is about 20 and $8 \mathrm{~dB}$ on average in the frequency range of $f=0.094$ to $f=0.124$ and $f=0.17$ to $f=0.24$, respectively, and these stopbands cover the first and second blade passing frequencies.

\section{Performance of a three-dimensional prototype with four membranes}

It is known that a fan radiates sound in all the circumferential directions, but a tensioned membrane cannot be installed circumferentially. To tackle this problem, a prototype of four membranes covered with one cylindrical cavity housing an axial fan is proposed. As shown in Fig. 5(a), four membranes with the length $L=300 \mathrm{~mm}$ and width of $h=100 \mathrm{~mm}$ are covered by a cylindrical cavity with radius $R=200 \mathrm{~mm}$ and results in an expansion ratio of 12.6. The duct has a cross-section of $100 \times 100 \mathrm{~mm}$. The membrane has a mass ratio of $m=1.4$ and a tension of $T=0.2$.

The axial fan with the first blade passage frequency of $400 \mathrm{~Hz}$ (normalized frequency $f=0.1$ ) is installed. Figure 5(b) shows the SPL measured with the extraction of the dipole component for the straight duct (dashed line) and that for the four membranes with cylindrical cavity (solid line) when the fan is installed at the center of the test rig. The SPL is decreased with the sequence of blade passage frequencies for the straight duct without silencing treatment (dashed line). The SPL at the second and third blade passage frequencies is much lower than that at the first blade passage frequency by about $25 \mathrm{~dB}$ and $50 \mathrm{~dB}$, respectively. Figure 5(c) shows the comparison between the measured $\mathrm{IL}_{\mathrm{d}}$ and the numerical predicted IL in the $3 \mathrm{D}$ configuration. The agreement between them is quite good regarding the overall IL level and the spectral pattern. It shows that the fan noise is suppressed significantly by about $40 \mathrm{~dB}$ at the first BPF of $f=0.117$ and by $20 \mathrm{~dB}$ at the second BPF of $f=0.233$.

\section{ANALYSIS OF THE PERFORMANCE AND ANTI-SYMMETRICAL EFFECT}

Aiming for an effective fan noise control device that works preferably in the broadband covering first and second blade passage frequency, three issues are addressed at the design stage. First, the effect of various design parameters on the performance of the present device should be identified such as the cavity shape, the membrane-to-air mass ratio, and the tension of the membrane. The second issue is the effect of mean flow on the sound radiation from the axial fan of a dipole nature and the influence of noise suppression by the membrane when the mean flow passes over it. The third issue is a search for the location of the axial fan such that the most effective noise suppression can be obtained.
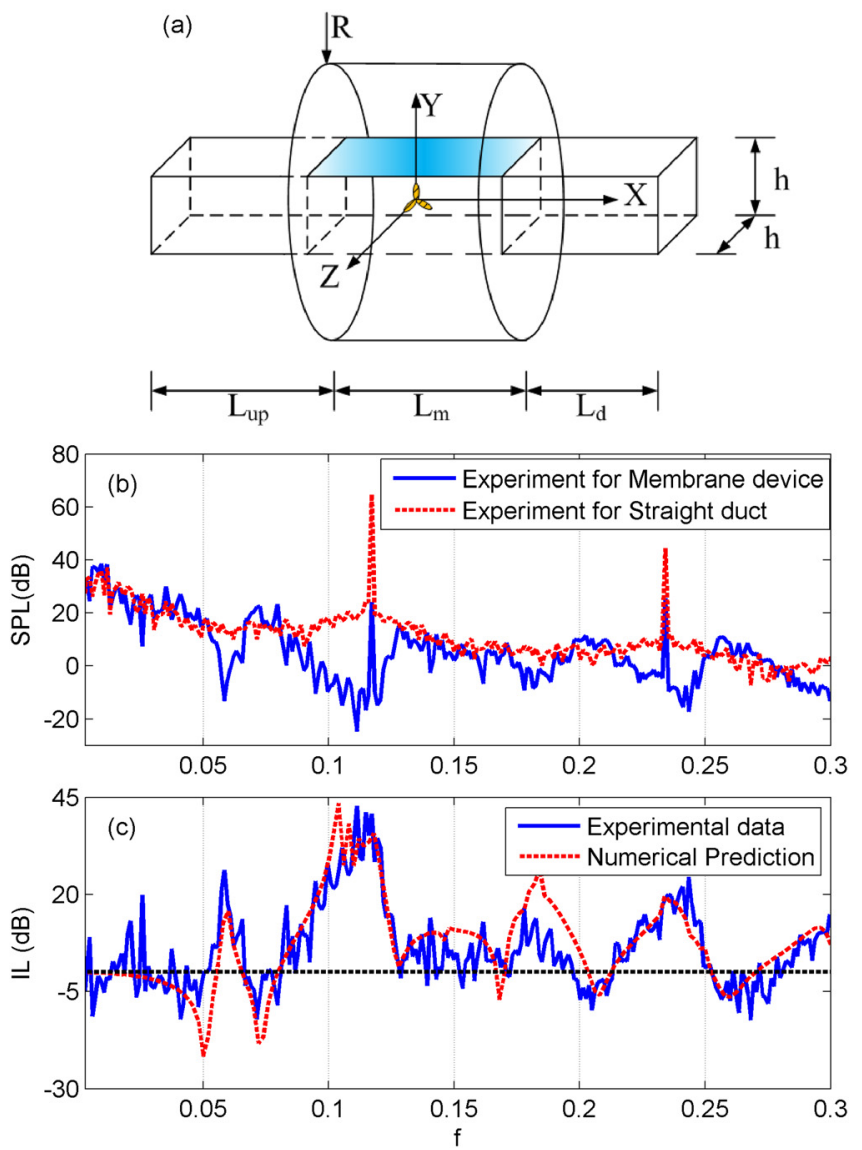

FIG. 5. (Color online) Performance of four-membrane silencer. (a) Prototype of the four-membrane silencer. (b) SPL spectra at the exit of the duct for the four membrane silencer. (c) Performance comparison of the measured $\mathrm{IL}_{d}$ (solid line) and numerical prediction (dashed line). 

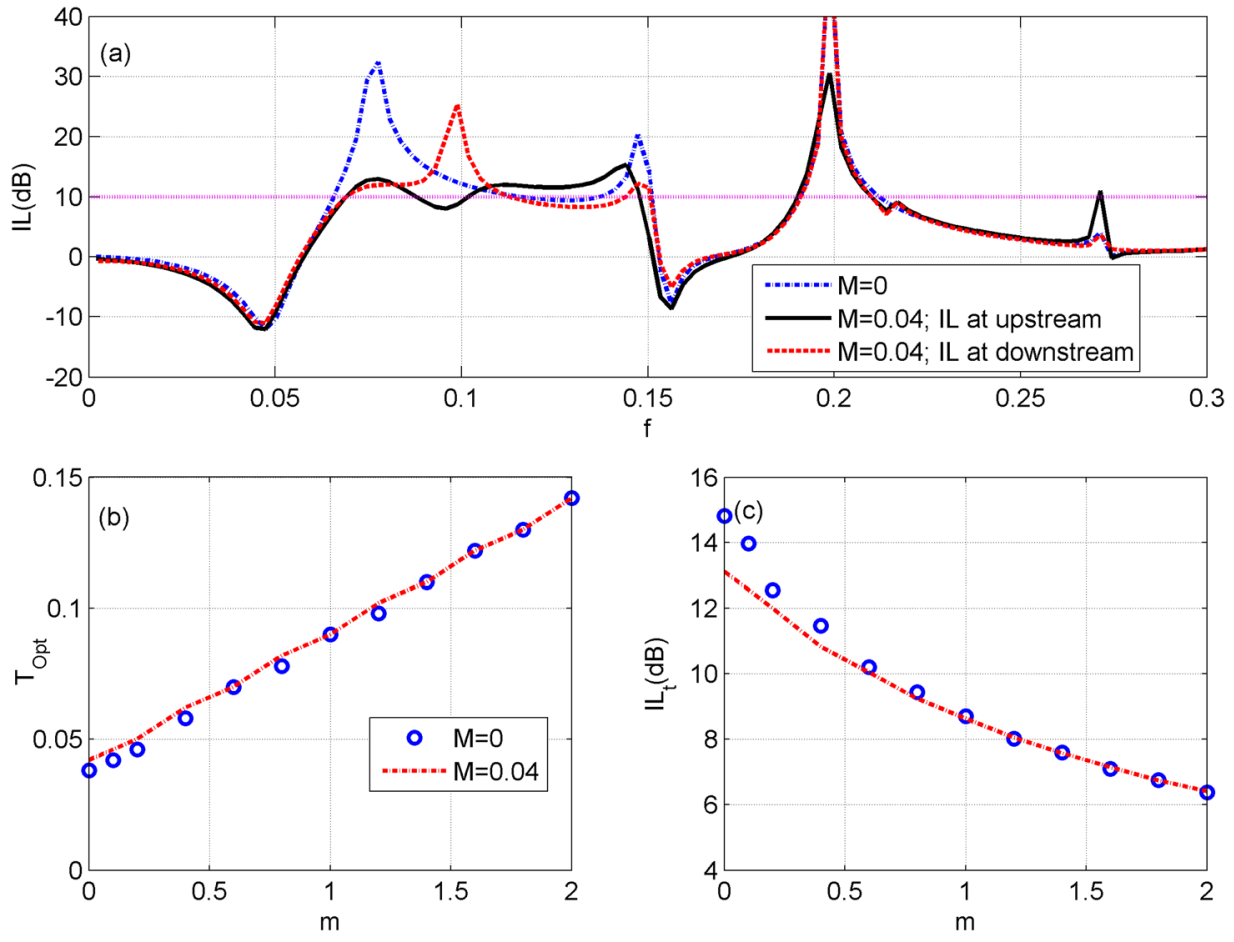

\section{A. Optimization of the performance}

To evaluate the performance of the proposed device, the total IL in the specified frequency range of interest, $f_{r}$ $=\left[f_{a}, f_{b}\right]$ is defined and calculated by

$$
\mathrm{IL}_{t}=10 \log _{10} \frac{\left|\int_{f_{a}}^{f_{b}} \int p_{t}^{2} d s d f\right|_{\text {original }}}{\left|\int_{f_{a}}^{f_{b}} \int p_{t}^{2} d s d f\right|_{\text {silenced }}}
$$

where $p_{t}^{2}=p_{\text {upexit }}{ }^{2}+p_{\text {downexit }}{ }^{2}$, and the subscripts "original" and "silenced" represent the use of a straight duct and the proposed device, respectively.

The axial fan used for the experimental validation is the modified version of the fan taken from the market. The fan has seven rotor blades and four downstream struts. In order to obtain the ideal feature of the dipole for the ducted fan in the current study, the most effective and simplest way is to make the same number of blades and struts so that the spinning pressure mode vanishes and, as a result, the unsteady thrust force component radiates the noise most efficiently and the drag force vanishes (Huang, 2003). The number of downstream struts is modified to 7 . The designed operational speed is in the range of 2500 to $3950 \mathrm{rpm}$ with the first BPF of 250 to $500 \mathrm{~Hz}$ (normalized frequency $f_{a}=0.073$ to $f_{b}=0.145$ ). Then the performance of this device is optimized by searching for the optimal structural parameters in order to obtain the maximum total IL in this frequency range. In the current study, the geometry of the configuration is fixed at $L=3$ and $h_{c}=2$ and both ends of the ducts are assumed to be infinite. The results of the performance optimization are shown in Fig. 6. Figure 6(a) compares the IL spectra with the optimized parameters $T=0.058$ and $m=0.4$ at $M=0$ (dashed dotted line) and $M=0.04$ upstream (solid line) and $M=0.04$ downstream (dashed line). Two sharp peaks in the
FIG. 6. (Color online) Optimization results. (a) Optimized spectral pattern with fixed tension and mass $(m=0.4$ and $T=0.058$ ) at different flow speeds. (b) Optimal tension. (c) Total insertion loss as a function of $m$. frequency range of $f=0.074$ to $f=0.147$ are observed at $M=0$ or $M=0.04$. The IL spectrum appears to be more flattened and the peak location is changed when there is flow. Apparently there is slight degradation of the performance. Figures 6(b) and 6(c) display the optimal tension $T_{\mathrm{opt}}$ and total $\mathrm{IL}_{t}$ as a function of $\mathrm{m}$ for flow speeds $M=0$ and $M=0.04$, respectively. Figure 6(b) suggests that optimal tension $T_{\mathrm{opt}}$ increases monotonically with the membrane mass $m$ when there is no flow and flow condition. As shown in Fig. 6(c), when the mass of the membrane increases, the maximum achievable $\mathrm{IL}_{t}$ decreases gradually. In order to have total $\mathrm{IL}_{t} \geq 10 \mathrm{~dB}$ without exceeding the fatigue stress of the material used for the thin membrane, the optimal mass of the membrane is found to be in the range of $0.2 \leq m \leq 0.6$. Two observations are made here. First, the flow passage over the membrane affects the performance so long as the membrane mass is low enough $(m<0.6)$. Second, when air flow passes over the membrane and deforms it, a slight increment of tension is required to overcome the deterioration and good performance can still be roughly maintained to have $\mathrm{IL}_{t}$ of $10 \mathrm{~dB}$. The optimal result reflects that there is a delicate coupling between the membrane dynamic and the fluidsound field in the frequency range of interest.

\section{B. Mean flow effect}

With the membrane mass $m=0.4$ and $T=0.05$ and source being located at center $x_{s}=0$, the flow effect on the performance of the present noise control device is investigated here. Two sharp peaks (dashed dotted line) shown in Fig. 7(a) represent the reference case $M=0$. When there is flow, the levels of the two peaks decrease and they are also smoothed out. Apart from this, the IL between the first and second peaks undergoes slight changes with the flow. The first peak of the IL downstream shifts to a higher frequency and this spectral change reflects that the sound-membrane 

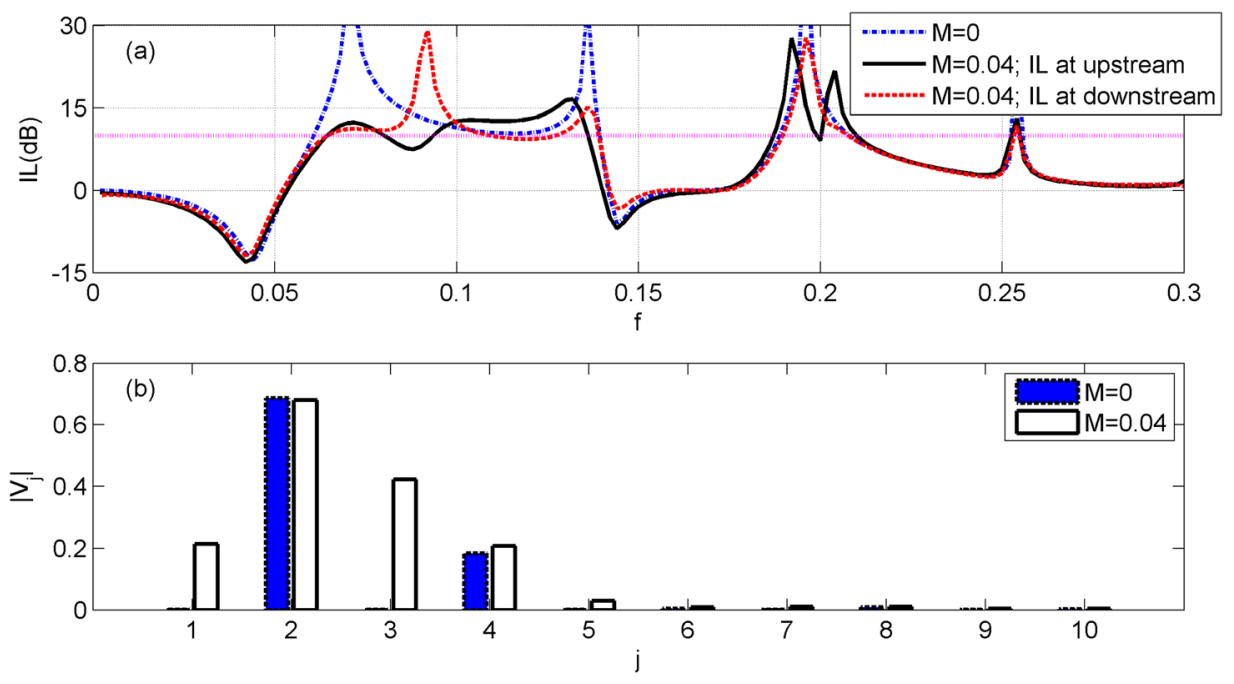

FIG. 7. (Color online) Modal analysis for two different flow speeds $M$ $\left(x_{\mathrm{s}}=0, m=0.4, T=0.05\right)$. (a) IL performance. (b) The velocity amplitude of each mode at $f=0.09$. (1a)-(1d) Modal amplitudes at different frequen$\operatorname{cies} f$.
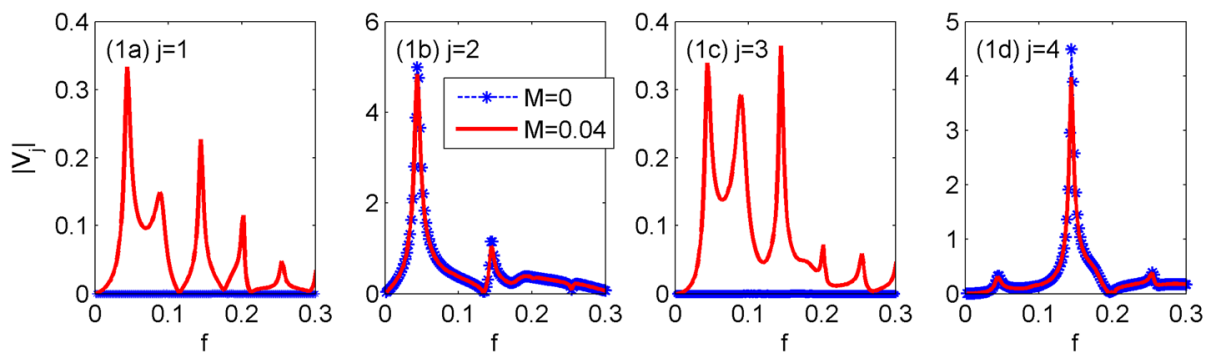

interaction is affected due to the flow. In order to understand the spectral change, the modal response of the membrane is analyzed. The membrane vibration is decomposed into axial modes of in vacuo membrane vibration. Figure 7(b) depicts the velocity amplitude of each mode $\left|V_{j}\right|$ at one particular frequency $f=0.09$. When there is no flow, only even modes are excited due to the symmetrical nature of the dipole sound source. As flow speed increases, other vibration modes are induced, especially the first and third modes. In order to further understand the influence of the odd mode in each frequency when there is flow, the vibration amplitude spectra

of each individual mode $V_{j}$ for flow conditions is investigated and they are displaced from Figs. 7(1a) to 7(1d). Generally speaking, the vibration amplitudes of $\left|V_{1}\right|$ and $\left|V_{3}\right|$ in the case of $M=0.04$ are much higher than those without flow. The results imply that when the air flow passes over the membrane, it significantly excites the first and third mode of membrane into vibration.

The physical mechanism can be further analyzed by the modal radiation impedance and kinematic boundary condition on the surface of the membrane. Table I lists the modal radiation impedance of the first four orders at $f=0.09$.

TABLE I. Modal radiation impedance of $f=0.1$ in the duct when $M=0$ and 0.04 .

(a) Radiation impedance in the duct $\left(U=0, x_{s}=0\right)$

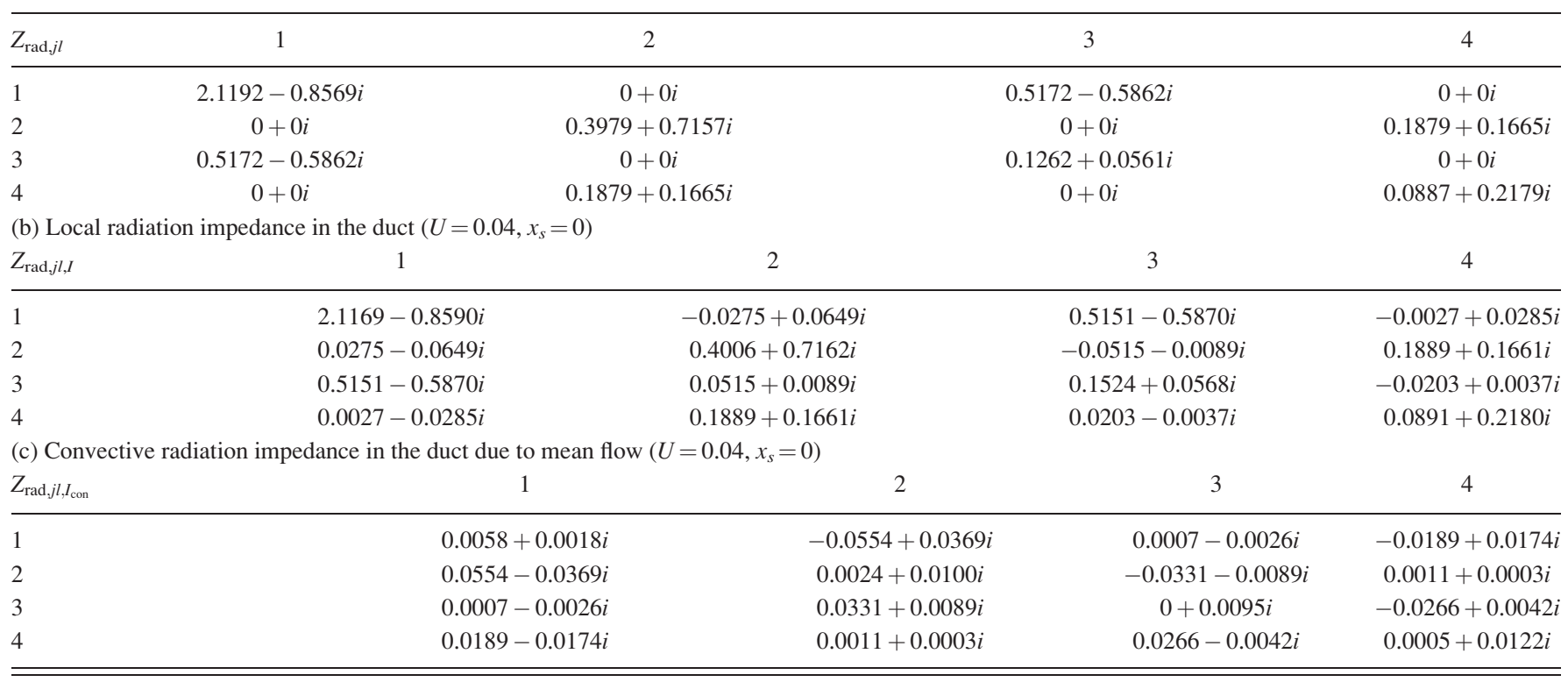




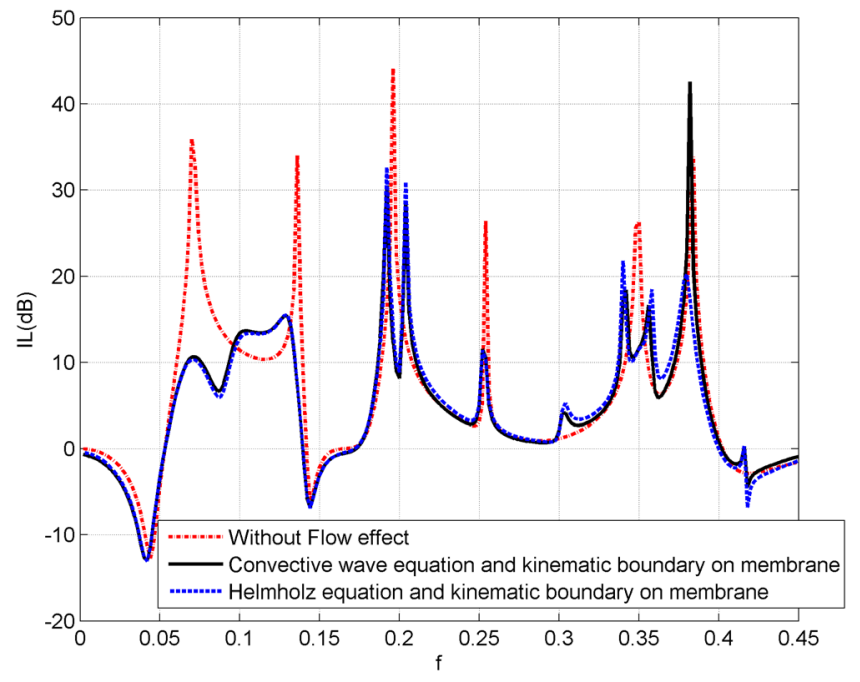

FIG. 8. (Color online) IL spectrum for different acoustic domain and boundary conditions for the flow effect.

When $M=0$, the total modal impedance in the duct is perfectly symmetrical and the odd-even modes are fully decoupled with each other as listed in Table I(a). The modal impedance consists of two parts: the local impedance and the convective impedance as indicated in Eq. (5). The convective impedance appears so long as there is flow. Notice that a strong cross-modal interaction appears in both terms as listed in Tables I(b) and I(c) when there is flow, which causes the whole matrix to become asymmetrical. This cross-modal interaction is attributed to the comprehensive effect of the flow, which can spread over many in vacuo modes.

In regard to the flow induced modes, two different approaches for the flow effect on the FEM model are investigated. (1) For the acoustic domain, the fluid in the duct is governed by the convection wave equation with acoustic
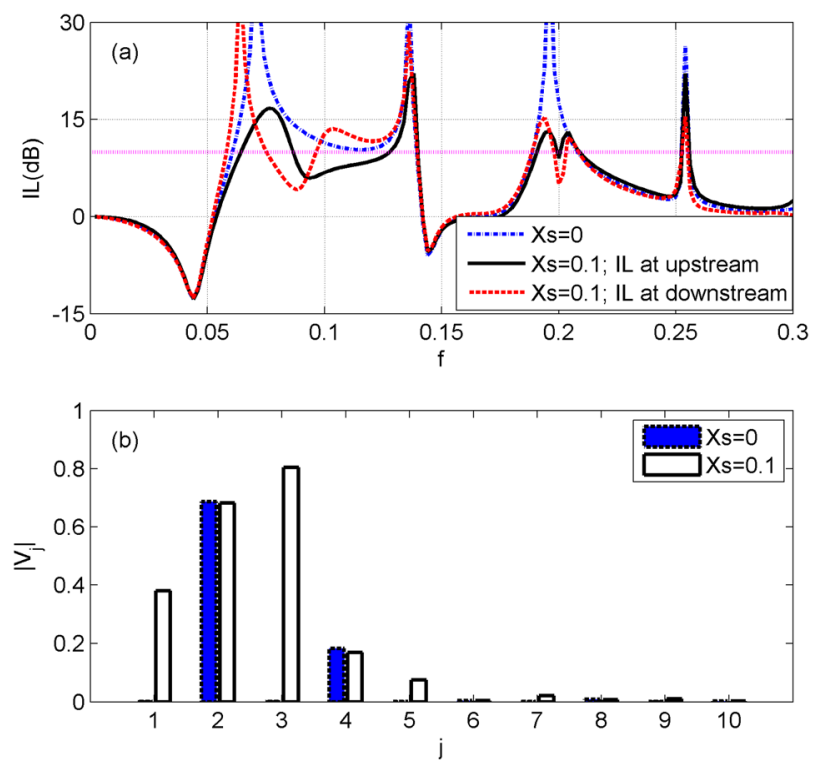

FIG. 9. (Color online) Modal analysis for sound source position for the flow speed $M=0, m=0.4$, and $T=0.05$. (a) IL performance at two outlets of the duct. (b) The velocity amplitude of each mode at $f=0.09$.

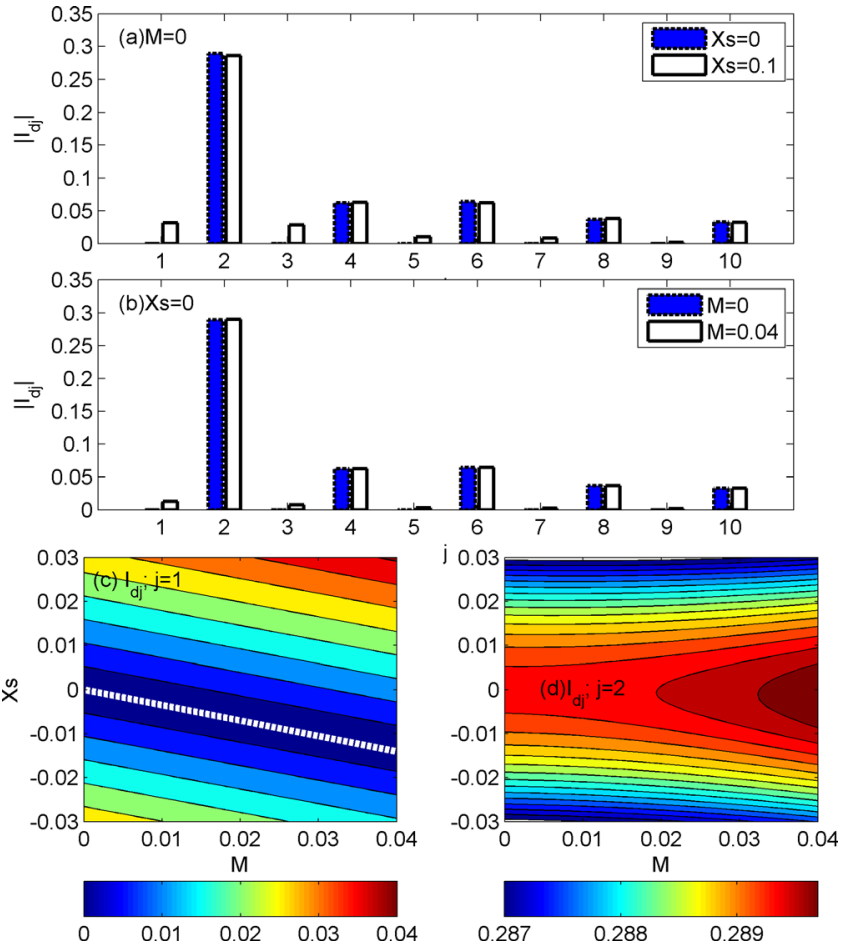

FIG. 10. (Color online) Pressure loading distribution from the dipole source at $f=0.09$. (a) Modal excitation amplitude by dipole source at $M=0$ for different source locations. (b) Modal excitation amplitude by dipole source at $x_{s}=0$ for different Mach numbers. (c) Excitation of the first mode as a contour of $M$ and $x_{s}$. (d) Excitation of the second mode as a contour of $M$ and $x_{s}$.

pressure coupled by a kinematic boundary condition on the membrane surface. (2) The fluid in the duct is governed by the acoustic wave equation without flow but the kinematic boundary condition is considered on the membrane surface. The comparison of IL performance of the device under these two approaches for flow effect is shown in Fig. 8. These two curves are generally similar but a slight difference is observed at very high frequency. This means that the flow effect on the convective wave equation is not influential on the performance of the proposed device. However, its effect regarding the kinematic boundary condition on the membrane is very important. Due to the coupling with the kinematic boundary, the displacement $\eta$ of the membrane at odd mode is excited by the differential equation of $\partial \eta / \partial x$ in the kinematic boundary and hence the sound radiation and IL are also affected.

\section{Influence of sound source position}

The odd modes can be induced significantly due to the kinematic boundary condition when there is flow. In addition, the different wave speeds due to the flow at the two sides of the sound source result in the asymmetrical loading on the membrane. The unbalanced loading can also be obtained directly by placing the source deviated from the center without flow. Figure 9(a) shows the IL spectrum for the sound source at different locations when $M=0$. The IL for the dipole source at the center $x_{s}=0$ is represented by a dashed dotted line, while the IL at the upstream exit and that at the downstream exit when $x_{s}=0.1$ are represented by a 
solid line and dashed line, respectively. The behavior of peak smooth of the IL when $x_{s}=0.1$ is similar to the way of increasing flow speed when the dipole source is located at center $x_{s}=0$. As shown in Fig. 9(b), the odd mode vibration amplitude is highly enhanced for $f=0.09$ when $x_{s}=0.1$ and is compared with $x_{s}=0$. Hence, the sound radiation from the membrane at these odd modes is significantly increased and this eventually degrades the silencing performance of such device.

In principle, the response of the membrane depends on the pressure loading distribution on it. One of the loadings is the dipole source excitation. It is now re-examined as a sum of a series of in vacuo modes [see Eq. (8)] at different source positions and flow speeds $M$. Figures 10 (a) and 10(b) demonstrate the amplitude of the first ten modal excitations from the dipole source at $f=0.09$ at different source positions $x_{s}$ when $M=0$ and at different flow speeds $M$ when $x_{s}=0$, respectively. Both results indicate that the odd modes can be excited either by shifting the source position or by introducing flow effect. Figures 10(c) and 10(d) show the amplitude of the first and second mode of the excitation pressure from the dipole source, respectively, as a function of both source location and mean flow speed. As shown in Fig. 10(c), the thick dashed line represents zero excitation pressure from the dipole source for the first mode. Focusing on this line, when the flow speed increases, the excitation of the first mode tends to vanish with a more negative source position. This result is due to the convective effect on the sound speed towards the downstream direction, which accelerates the sound propagation. However, a deceleration effect is imposed towards the upstream direction. Then, in order to achieve the balance, the sound source has to be shifted upstream due to the different sound speeds at the two sides of the axial fan. The slope of the dashed line is roughly related to the membrane length $L$. For example, when $L=3$ and $f=0.09$, the source location can be approximately shifted by the value of $\left|\Delta x_{s} / \Delta M\right|$ which is proportional to $L$. On the contrary, the second modal excitation from the dipole source always exists in the whole calculated range of $x_{s}$ and $M$ as shown in Fig. 10(d). Therefore, the explanation is consistent with the results in Figs. 10(a) and 10(b), which also consolidates the main findings.

\section{CONCLUSIONS}

Comprehensive analyses have been conducted for the purpose of understanding the sound-membrane coupling mechanism for an axial flow fan inside a tensioned membrane silencing device with mean flow effect. The performance of the cavity-backed tensioned membrane in controlling subsonic axial fan noise has been investigated analytically, numerically, and validated by experiments. The optimization study was also performed on several parameters such as the tension and mass of the membrane in order to determine the maximum total IL in a specified frequency range. The major conclusions that can be drawn follow.

(1) A two-dimensional analytical formulation and threedimensional numerical model have been established to evaluate the performance of the proposed device with tensioned membrane covered by a cavity to control noise radiated from a subsonic axial flow fan. With a suitable tension in the axial direction to balance the deformation when there is flow, the proposed device with an expansion ratio of 3 can achieve a total IL of $10 \mathrm{~dB}$ over a frequency range of interest that can cover the blade passing frequencies of the fan with various rotational speeds.

(2) The qualitative effect of the flow on the proposed tensioned membrane housing performance is the influence of the symmetrical feature of the dipole source. When there is flow, the odd mode vibration of the membrane is excited by the even mode of the kinematic boundary on the membrane. As a result, asymmetrical excitation force and the strong cross-modal coupling in the modal radiation impedance are found due to the convective effect.

(3) An asymmetrical feature can also be observed by offsetting the dipole source away from the center of the membrane in the duct. This is caused by the unbalanced acoustic pressure loading on the membrane. Such an offcenter arrangement of the dipole source can balance the asymmetrical behavior resulting from the flow effect.

(4) Experimental studies were conducted on two devices. One comprised two-tensioned membranes with two rectangular cavities to verify the numerical and analytical model. There was satisfactory agreement between them. With a tension of $300 N$, the proposed device with an area expansion ratio of 5 could reduce the noise radiation of an axial flow fan by about 20 and $5 \mathrm{~dB}$ for the first and second blade passing frequencies, respectively. The other was a prototype made of a cylindrical cavity covered with four-tensioned membranes enclosing the subsonic axial fan with an expansion ratio of 12.6. With a tension of $220 \mathrm{~N}$, the measured result demonstrates that there is a significant noise reduction of about $40 \mathrm{~dB}$ at the first blade passing frequency of the fan, thus showing the promises of the device to control fan noise directly.

\section{ACKNOWLEDGMENTS}

The project is funded by General Research Grant from the Hong Kong SAR government (B-Q33E).

Atig, M., Dalmont, J. P., and Gilbert, J. (2004). “Termination impedance of open ended cylindrical tubes at high sound pressure level," C. R. Acad. Sci. Paris, Series II 332, 299-304.

Baumeister, K. J. (1979). "Evaluation of optimized multisectioned acoustic liners," AIAA J. 17, 1185-1192.

Beranek, L., and Ver. I. (2006). Noise and Vibration Control Engineering: Principles and Applications (Wiley, Hoboken, NJ), pp. 279-343.

Choy, Y. S., and Huang, L. (2002). "Experimental studies of a drumlike silencer," J. Acoust. Soc. Am. 112, 2026-2035.

Choy, Y. S., and Huang, L. (2005). "Effect of flow on the drumlike silencer," J. Acoust. Soc. Am. 118, 3077-3085.

Curle, N. (1955). "The influence of solid boundaries on aerodynamic sound," Proc. R. Soc. London, Ser. A 231, 505-514.

Doak, P. E. (1973). "Excitation, transmission and radiation of sound from source distributions in hard-walled ducts of finite length (I): the effects of duct cross-section geometry and source distribution space-time pattern," J. Sound Vib. 31, 1-72.

Envia, E. (2001). "Fan noise reduction: An overview," NASA/TM No. 2001-210661.

Eversman, E. (2001). "The boundary condition at an impedance wall in a non-uniform duct with potential mean flow," J. Sound Vib. 246, 63-69. 
Ffowcs Williams, J. E., and Hawkings, D. L. (1969). "Sound generation by turbulence and by surfaces in arbitrary motion," Philos. Trans. R. Soc. London, Ser. A 264, 321-342.

Gerard, A., Berry, A., Masson, P., and Gervais, Y. (2009). "Modelling of tonal noise control from subsonic axial fans using flow control obstruction," J. Sound Vib. 321, 26-44.

Gerard, A., Berry, A., Masson, P., and Moreau, S. (2012). "Semi-active method to control tonal noise from fans," Internoise, August 19-22, 2012, New York.

Gerhold, C. H. (1997). "Active control of fan generated tone noise," AIAA J. 35, 17-22.

Gutin, L. (1948). "On the sound field of a rotating propeller," Zh. Tekh. Fiz. 6, 899-909.

Huang, L. (2003). "Characterizing computer cooling fan noise," J. Acoust. Soc. Am. 114, 3189-3200.

Huang, L., Ma, X., and Feng, L. G. (2010). "Suppression of broadband noise radiated by a low-speed fan in a duct," J. Acoust. Soc. Am. 128, 152-163.

Kameier, F., and Neise, W. (1997). "Experimental study of tip clearance losses and noise in axial turbomachinery and their reduction," Trans. ASME J. Turbomach. 119, 460-471.

Koopmann, G. H., Fox, D. J., and Neise, W. (1988). "Active source cancellation of the blade tone fundamental and harmonics in centrifugal fans," J. Sound Vib. 126, 209-220.

Kuttruff, H. (2000). Room Acoustics (E \& FN Spon, New York), pp. 59-71.

Lauchle, G. C., Mac Gillivray, J. R., and Swanson, D. C. (1997). "Active control of axial-flow fan noise," J. Acoust. Soc. Am. 101, 341-349.

Lighthill, M. J. (1952). "On sound generated aerodynamically. I. General theory," Proc. R. Soc. London, Ser. A 211, 564-587.
Liu, Y., Choy, Y. S., Huang, L., and Cheng, L. (2012). "Noise control of a dipole source by the silencer with tensioned membranes," J. Acoust. Soc. Am. 132, 1392-1402.

Maa, D. Y. (1998). "Potential of microperforated panel absorber," J. Acoust. Soc. Am. 104, 2861-2866.

Munjal, M. L. (1987). Acoustics of Ducts and Mufflers (Wiley, New York), pp. 42-103.

Nijhof, M. J. J., Wijnant, Y. H., Boer, A. D., and Beltman, W. M. (2004a). "Optimizing circular side-resonators to reduce computer fan noise," Noise-Con, July 12-14, Baltimore, MD.

Nijhof, M. J. J., Wijnant, Y. H., Boer, A. D., and Beltman, W. M. (2004b). "Reduction of fan noise by means of (circular) side-resonators: theory and experiment," International Conference on Noise and Vibration Engineering, ISMA, September 20-22.

Parker, R. (1972). "The effect of the acoustic properties of the environment on vibrations of a flat plate subject to direct excitation and to excitation by vortex shedding in an airstream," J. Sound Vib. 20(1), 93-112.

Rodarte, E., and Miller, N. R. (2001). "Flow induced noise in heat exchangers," Report No, ACRC-CR42, pp. 35-56.

Walker, B. E., Hersh, A. S., Heidelberg, L. J., Sutliff, D. L., and Spencer, M. E. (1999). "Active resonators for control of multiple spinning modes in an axial flow fan inlet," AIAA J. 99-1853, 339-348.

Wu, M. Q. (1997). "Micro-perforated panels for duct silencing," Noise Control Eng. J. 45, 69-77.

Ville, J. M., and Richard, J. S. (1980). "Experimental investigation of the radiation of sound from an unflanged duct and a bellmouth including the flow duct," NASA technical paper No. 1697. 